\title{
Atención Primaria de Salud: Factores de Desmotivación y Estabilidad Laboral de Médicos Generales
}

\author{
Fabiola Ríos Heldta ${ }^{a}$, Luisa Schonhaut Berman ${ }^{\mathrm{b}}$
}

\begin{abstract}
a Médico. Magíster en Salud Pública, Universidad de Chile. Medico Asesor, Dirección del Servicio de Salud Metropolitano Central, Santiago, Chile.

${ }^{\mathrm{b}}$ Medico. Magíster en Salud Publica, Magíster en Educación para la salud. Clínica Alemana, Universidad del Desarrollo, Chile.
\end{abstract}

Correspondencia: Fabiola Ríos Heldt. Dirección del Servicio de Salud Metropolitano Central, Santiago,

Chile. Dirección postal: Chile España 353, depto. 705. Nuñoa. Correo electrónico: riosmed@gmail.com.

Recibido el 21 de julio de 2009.

Aceptado para su publicación el 12 de septiembre de 2009.

\section{RESUMEN}

Objetivo. Conocer las motivaciones laborales de los médicos chilenos con mayor permanencia en Atención Primaria de Salud (APS).

Metodología. Estudio cuanti-culitativo descriptivo. Se reunió información biodemográfica y laboral de médicos chilenos que trabajaban en 10 de 15 establecimientos de APS de un Servicio de Salud urbano (Región Metropolitana). Siete médicos con mayor estabilidad laboral, es decir, permanencia superior al promedio, y 2 directivos fueron entrevistados en profundidad. Los resultados fueron sometidos al juicio de expertos.

Resultados. Se identificaron 21 médicos chilenos, que cubrían un tercio de las horas médicas totales. La mitad de estas horas eran cumplidas por los de mayor antigüedad. La permanencia promedio fue 8,5 años. Los profesionales con mayor estabilidad laboral fueron significativamente mayores (44 vs. 28 años) y habían ejercido cargos directivos. Destacaron las motivaciones trascendentes, como la vocación, relación entablada con pacientes y comunidad y el compromiso con la salud pública. Entre las propuestas sugeridas para el reclutamiento de médicos destaca el desarrollo profesional, mientras que las estrategias para combatir la monotonía favorecerían su estabilidad laboral.

Conclusiones. Este estudio permite una aproximación a las motivaciones que llevan al médico chileno a trabajar en forma estable en APS. Parece fundamental tener una visión más holística que la sola atención curativa individual y la resolución de contingencias, promoviendo estrategias que permitan al profesional trascender e impactar en la salud de la población.

Palabras clave. Médicos. Atención Primaria. Motivación. Trabajadores.

\section{ABSTRACT}

Primary Healthcare: De-motivation factors and Work stability of Primary care physicians.

Objective. To determine Chilean physicians' motivation for longer service in Primary Health Care (PHC).

Methodology. Semiquantitative study of the demographic data of Chilean physicians working at 10 of 15 PHC centers in a Metropolitan area. High job stability was defined as an above average length of service. Seven physicians that were judged as "highly stable" according to this definition and two administrators took part in a detailed interview for the qualitative study. Results were submitted for subjective evaluation by a panel of experts.

Results. The sample was made up of 21 physicians, the average length of service was 8.5 years. Physicians who had worked in PHC for a longer period of time were older (44 vs 28 years) and had performed administrative duties. They listed motivations such as vocation, physician-patient and physician-community relationship and commitment to public health. Amongst the proposals suggested in order to recruit PHC physicians and ensure job stability were opportunities for professional development, and strategies to avoid monotony.

Conclusions. This study helps to determine what motivates Chilean physicians to remain in PHC for longer periods of time. A more holistic view of patient care, including direct patient contact appears to be needed, as well as providing an opportunity to participate in solving community problems. Key words. Physicians, Primary Care, Motivation, Workers.

\section{INTRODUCCIÓN}

A partir de la declaración de Alma Ata1 ${ }^{1}$, formulada hace 30 años, se han desarrollado crecientes esfuerzos y políticas a nivel internacional para el fortalecimiento de la Atención Primaria (APS) como estrategia fundamental en salud². Siguiendo la tendencia mundial, recientemente se implementó en nuestro país, Chile, la mayor reforma de salud, desde la creación del Servicio Nacional de Salud, otorgando un rol protagónico a la APS, bajo el modelo de salud familiar ${ }^{3}$. 
En Chile se estima un significativo déficit de médicos en APS, como reflejo de una importante insuficiencia del sistema, en relación a la capacidad de reclutamiento y retención del recurso humano, en especial del recurso médico, a este nivel ${ }^{4}$. Los médicos, insatisfechos con su actividad laboral, presentan una alta rotación ${ }^{5-6}$, sin lograr consolidarse en los equipos de salud.

La insatisfacción laboral podría atribuirse a la crisis de la práctica médica ${ }^{7}$, desencadenada por los cambios ocurridos en el entorno profesional ${ }^{8}$, y que se asocian a una mayor complejidad de las enfermedades, aumento de la oferta tecnológica para el diagnóstico y tratamiento, incrementos de los costos en salud y judicialización de la medicina. Frente a esta realidad el médico ha debido asumir una creciente responsabilidad clínica y administrativa, pero con una baja autonomía en la toma de decisiones ${ }^{9}$ y capacidad resolutiva ${ }^{10-12}$. A esto habría que agregar la falta de oportunidades de desarrollo profesional, expectativas insatisfechas e inadecuados incentivos ${ }^{13-15}$.

La cara opuesta de esta moneda estaría dada por la especialización médica, alternativa que representa un nicho laboral atractivo, prestigio, mejor calidad de vida y bienestar económico ${ }^{16-17}$. Por este motivo, para muchos de los facultativos chilenos la motivación de incorporarse a la APS se relacionaría con la opción de reunir los requisitos que le permitan postular a programas de formación de especialistas ${ }^{18}$.

La motivación puede ser entendida como la fuerza impulsora de la conducta. De acuerdo a la teoría de
Maslow $^{19}$, esta fuerza tiende a satisfacer las necesidades humanas, ubicándose en la base las necesidades fisiológicas y en niveles superiores las de autorrealización.

Cuando llevamos la motivación al terreno del mundo laboral, podemos basarnos en la teoría motivacional de Pérez-López ${ }^{20}$, quien clasifica la motivación dependiendo de su origen en tres tipos:

1. La Motivación Extrínseca se relaciona con las recompensas, que pueden ser materiales, como el salario, o no materiales, como el reconocimiento de los pares o superiores.

2. La Motivación Intrínseca alude a la satisfacción que la persona consigue por la realización de su trabajo y satisface una necesidad psicológica.

3. La Motivación Trascendente se refiere a las consecuencias que van a tener en otras personas, las decisiones que el profesional tome durante su ejercicio profesional, satisfaciendo necesidades vocacionales $y$ afectivas.

El objetivo del estudio es identificar las motivaciones laborales de los médicos chilenos con mayor permanencia en APS de un Servicio de Salud urbano de la Región Metropolitana.

\section{MATERIAL Y MÉTODO}

El presente es un estudio cuanti-cualitativo, descriptivo, desarrollado durante los meses de marzo a agosto del 2008 en un Servicio de Salud de la Región Metropolitana.

Dicho Servicio de Salud cuenta con 18 centros de Sa-

\begin{tabular}{ccccccc}
\hline Médicos & Sexo & Edad & $\begin{array}{c}\text { Tipo de } \\
\text { contrato }\end{array}$ & $\begin{array}{c}\text { Tipo de } \\
\text { egreso (años) }\end{array}$ & $\begin{array}{c}\text { Universidad } \\
\text { de egreso }\end{array}$ & $\begin{array}{c}\text { Antigüedad } \\
\text { en APS }\end{array}$ \\
\hline M1 & H & 38 & Indefinido & 10 & Universidad de Chile & 10 \\
M2 & M & 45 & Indefinido & 18 & Universidad de Chile & 18 \\
M3 & H & 49 & Indefinido & 25 & Universidad de Chile & 25 \\
M4 & M & 41 & Indefinido & 15 & Universidad de la Frontera & Universidad de Chile \\
M5 & M & 42 & Indefinido & 8 '5 & Universidad de Chile & 15 \\
M6 & H & 37 & Indefinido & 10 & Universidad de Chile & 15 \\
\hline
\end{tabular}

Tabla 1. Perfil de los Médicos entrevistados.

\begin{tabular}{cccc}
\hline Directores & Sexo & Tiempo en el Cargo & Profesión \\
\hline D1 & F & 5 años & Enfermero \\
D2 & M & 5 años & Matrona \\
\hline
\end{tabular}

Tabla 2. Perfil de los Directivos entrevistados. 


\begin{tabular}{|c|c|c|c|}
\hline Aspecto & $\begin{array}{c}\text { Menor } \\
8 \text { años antigüedad }\end{array}$ & $\begin{array}{c}\text { Mayor o igual } \\
8 \text { años antigüedad }\end{array}$ & $\mathrm{p}$ \\
\hline Edad & Promedio 28,6 años (DS: 3,64 ) & Promedio 44 años (DS: 8.58) & $P<0.000$ \\
\hline Sexo & $45 \%$ Mujeres & $60 \%$ Mujeres & NS \\
\hline Estado civil & $88 \%$ Solteros & $50 \%$ soltero & NS \\
\hline Tipo de contrato & $\begin{array}{c}44 \% \text { Plazo fijo, } 22 \% \text { honorario, } \\
\text { 33,3\% Indefinido }\end{array}$ & $\begin{array}{c}50 \% \text { plazo fijo, } 10 \% \text { honorario, } \\
40 \% \text { indefinido }\end{array}$ & NS \\
\hline Horas semanales de trabajo & Promedio: 36.6 horas DS 9.52 & Promedio: 31,9 horas: DS: 14,15 & NS \\
\hline Otra actividad laboral & $44,4 \%$ & $60 \%$ & NS \\
\hline Hijos & $33,3 \%$ & $60 \%$ & NS \\
\hline Cargos directivos ejercidos & $0 \%$ & $50 \%$ & $P 0,013$ \\
\hline $\begin{array}{l}\text { Asistencia a capacitación promovida } \\
\text { por establecimiento en el ultimo año }\end{array}$ & $66,6 \%$ & $70 \%$ & NS \\
\hline
\end{tabular}

Tabla 3. Análisis cuantitativo de características biodemográficas y laborales.

\begin{tabular}{|c|c|c|}
\hline & Médicos & Directores \\
\hline $\begin{array}{l}\text { Motivaciones } \\
\text { extrínsecas }\end{array}$ & $\begin{array}{l}\text { Salario(0/8) } \\
\text { Contrato estable }(0 / 8) \\
\text { Reconocimiento de pares }(0 / 8)\end{array}$ & $\begin{array}{l}\text { Salario }(0 / 2) \\
\text { Contrato estable }(0 / 2) \\
\text { Reconocimiento de pares }(0 / 2)\end{array}$ \\
\hline $\begin{array}{l}\text { Motivaciones } \\
\text { intrínsecas }\end{array}$ & $\begin{array}{l}\text { Desarrollo profesional }(0 / 8) \\
\text { Desarrollo profesional }(0 / 2)\end{array}$ & Carrera funcionaria $(0 / 8)$ \\
\hline $\begin{array}{l}\text { Motivaciones } \\
\text { trascendentes }\end{array}$ & $\begin{array}{l}\text { Aporte a la Salud Pública }(7 / 8) \\
\text { Relación médico paciente }(8 / 8) \\
\text { Impacto en salud publica }(8 / 8)\end{array}$ & $\begin{array}{l}\text { Aporte a la Salud Pública }(2 / 2) \\
\text { Cercanía con las personas }(2 / 2) \\
\text { Compromiso con APS }(2 / 2)\end{array}$ \\
\hline $\begin{array}{l}\text { Rol formador de } \\
\text { la Universidad }\end{array}$ & $\begin{array}{l}\text { Rotaciones escasas en APS }(8 / 8) \\
\text { Cambios en la formación de } \\
\text { nuevas generaciones }(6 / 8)\end{array}$ & $\begin{array}{l}\text { Rotaciones escasas por APS (2/2) } \\
\text { Cambios en la formación de } \\
\text { nuevas generaciones (2/2) }\end{array}$ \\
\hline $\begin{array}{l}\text { Estrategias para } \\
\text { reclutamiento de médicos } \\
\text { en APS }\end{array}$ & Fomentar desarrollo profesional (8/8) & Fomentar desarrollo profesional (2/2) \\
\hline $\begin{array}{l}\text { Estrategias para } \\
\text { la permanencia de } \\
\text { los médicos en APS }\end{array}$ & $\begin{array}{l}\text { Necesidad de diversificar las } \\
\text { actividades para combatir } \\
\text { la monotonía }(8 / 8)\end{array}$ & $\begin{array}{l}\text { Necesidad de diversificar las } \\
\text { actividades para combatir } \\
\text { la monotonía (8/8) }\end{array}$ \\
\hline
\end{tabular}

Tabla 4. Síntesis de los diferentes énfasis en las motivaciones laborales para trabajar en APS.

lud, 15 de ellos corresponden a comunas urbanas. La población estimada para el año 2004 era de 723.056 habitantes, con un $60 \%$ de beneficiarios del sistema público de salud ${ }^{21}$.

En la fase cuantitativa se incluyeron todos los médicos chilenos que trabajan en 10 de los 15 establecimientos de APS urbanos. Se excluyeron aquéllos que cursaron los estudios de pregrado en el extranjero. La información sociodemográfica y laboral fue recopilada a partir de una base de datos proveniente de Di- recciones de Salud Municipal y se completó con una encuesta previamente validada.

A partir de la información obtenida, se identificaron los médicos chilenos con mayor estabilidad laboral, es decir, con una permanencia en APS superior al promedio, que fue 8,5 años. Mediante estadísticas descriptivas y analíticas (prueba de chi-cuadrado) se compararon las características biodemográficas y laborales de aquéllos con mayor antigüedad con los que tenían antigüedad inferior al promedio. 
Los médicos con una permanencia superior al promedio en APS fueron considerados como "casos ricos en información acerca de permanencia", y fueron estudiados mediante entrevistas en profundidad 22 . Además, se entrevistó a los directivos de dos establecimientos. Para el análisis de la información, los entrevistados se enumeraron arbitrariamente en forma correlativa. El perfil de los entrevistados se muestra en las tablas 1 y 2.

Las entrevistas, basadas en una pauta, fueron abiertas, flexibles, dinámicas y no estructuradas. En su totalidad fueron realizadas por las autoras. La definición del tamaño muestral se basó en el criterio de saturación o redundancia. La información recopilada en las entrevistas fue analizada de acuerdo a las recomendaciones de la investigación cualitativa ${ }^{23}$.

Con el intento de "mejorar el rigor científico", los resultados fueron sometidos al juicio de expertos en APS. Esta estrategia otorga credibilidad y disminuye el sesgo de los resultados de la investigación cualitativa. Los expertos fueron: un académico del departamento de medicina familiar y comunitaria de una prestigiosa universidad del país, un integrante de la directiva de la Sociedad de Medicina Familiar y un ex director de centro de salud de APS.

\section{RESULTADOS}

Se abordaron 10 Centros de Salud, correspondientes al $62,5 \%$ de los establecimientos de salud urbanos del Servicio de Salud en estudio. En estos centros trabajaban en el periodo entre 1 y 3 médicos chilenos, los que ejercían un tercio de las horas médicas totales (rango 18,0 a 49,6\%). De estas horas, el $49,15 \%$ eran realizadas por profesionales con mayor estabilidad laboral.

El total de médicos chilenos que trabajaba en dichos establecimientos fue 21 . Se obtuvo información biodemográfica y laboral de 19 (90,4\%). El promedio de permanencia en APS para el grupo estudiado fue de 8,5 años (rango 1 mes a 37 años).

En la tabla 3 se sintetizan los principales resultados obtenidos en el análisis cuantitativo; se comparan las características biodemográficas de los médicos con mayor permanencia en APS (definida como mayor al promedio de 8,5 años), con los más nuevos, destacando que los primeros eran significativamente mayores (44 versus 28 años) y habían ejercido cargos directivos. No hubo diferencia en cuanto a otras variables, como el sexo, estado civil, el tener hijos, las ho- ras semanales de trabajo en APS, el tipo de contrato o las oportunidades de capacitación.

En la tabla 4 se muestra la síntesis de los diferentes énfasis en las motivaciones laborales para trabajar en APS que se obtuvieron de las entrevistas realizadas. Estos resultados se muestran extensamente a continuación.

\section{1.- Motivaciones extrínsecas}

\subsection{Factor Económico}

A pesar de las reconocidas mejoras ocurridas en las remuneraciones, los entrevistados consideraron que éstas no son proporcionales a la carga laboral y responsabilidad asumida, por lo que consideraron que no sería un factor de motivación para el reclutamiento y permanencia en APS: "si bien los sueldos han mejorado en los últimos años, éste no es un factor motivacional" (M6), "el estímulo para incentivar a los médicos no es necesariamente económico, el estímulo va por otro lado" (D1). Las diferencias salariales de médicos entre distintos municipios podría gatillar cambios del lugar de trabajo dentro de la APS: "muchos médicos se van a comunas donde pagan mejor" (M7).

\subsection{Contratos estables}

La modalidad de contratación "a honorarios", característica del inicio del ejercicio profesional, fue asociada a una percepción de inestabilidad laboral e inseguridad: "aquí donde estoy, existe inestabilidad laboral, hay colegas que vienen recién llegando con contrato a honorarios" (M3), "no podemos ofrecer contratos más estables como indefinido o a contrata, acá ingresan a honorarios, no podemos competir con otras comunas o consultorios que dan más estabilidad" (D2).

\subsection{Reconocimiento de los pares}

Hubo coincidencia respecto a la percepción de prejuicio hacia los médicos de APS, ya sean generalistas o especialistas en Medicina Familiar.

"los especialistas piensan que somos malos médicos, que no nos alcanzó para especializarnos" (M7).

"es mal visto ser un médico de atención primaria" (M3)

"creo que aún está en el médico del nivel secundario, la visión de la baja calidad del médico de APS, eso les afecta" (D1)

\section{2.- Motivaciones intrínsecas}

\subsection{Desarrollo Profesional}

Los médicos percibían obstáculos para desarrollarse en APS, debido a que la política de los directivos estaría orientada a resolver la contingencia, tanto 
administrativa como asistencial: "a los directivos les importa más el rendimiento que tengamos, que darnos oportunidades de desarrollo profesional (M2), "el desarrollo profesional acá es limitado, no puedes ir a capacitaciones tan seguido como quisieras: que faltan médicos, que las vacaciones, que el invierno y uno se va posponiendo"(M1, M4, M7), "me he dado cuenta que la única forma de retenerlos por más tiempo es dándoles espacios para el desarrollo profesional" (D2).

\subsection{La opción por la especialización}

A pesar de que el interés inicial de algunos médicos era la especialización, manifestaron que, por diferentes motivos, debieron comenzar a trabajar en APS y posteriormente optaron por desarrollar ahí su carrera profesional: "cuando salí de la universidad iba a hacer especialidad, pero me gustó el trabajo en APS, entendí que lo hospitalario no era lo que buscaba"(M6), "los médicos buscan la especialidad para tener desarrollo económico y profesional a posterior" (D1).

\subsection{Carrera funcionaria}

Los médicos refirieron haber asumido cargos directivos por tiempos limitados, optando por la atención directa de pacientes, que es el aspecto que les generaría mayor satisfacción. Percibían los cargos técnicos como una oportunidad para modificar el modo de incrementar la calidad en las prestaciones de salud: "cargos directivos?..... sí he tenido, pero no, prefiero hacer clínica" (M1, M2, M4), "he sido jefe de programa y he asumido otros cargos técnicos, con el propósito de ser un aporte en la calidad de salud de la gente" (M1).

\section{3.-Motivaciones trascendentes}

\subsection{Relación Médico Paciente y su impacto en salud} Se atribuyó especial valoración a la atención integral, con enfoque biopsicosocial, no sólo en el aspecto curativo sino también promocional y preventivo: "en APS hay más cercanía con la gente" (M1), "me gusta la APS, me permite dar atención integral a la población" (M2).

\subsection{Compromiso con la Salud Pública}

Se resaltó la vocación y compromiso con la Salud Pública. Los directivos manifestaron que el médico chileno es un elemento valioso y recalcaron la necesidad de hacerlos parte de la toma de decisiones de su establecimiento: "creo que APS necesita buenos médicos y por eso estoy acá"(M7), "vocacionalmente, me siento muy satisfecho" (M3), "son pocos los médicos chilenos que se quedan, creo que su compromiso es real" (D1, D2).

\section{4.- Estrategias propuestas}

Las oportunidades de desarrollo profesional fueron destacadas unánimemente por los entrevistados como estrategia para el reclutamiento, aunque no necesariamente para la permanencia de los médicos que ya están trabajando motivados en APS: "en el último tiempo hubo oferta de especialización para médicos de APS. Eso está bien, pero para mi no es una motivación, me mueven otras motivaciones para estar en APS" (M1), "las becas de especialización para médicos de APS, son una buena forma y motivación para los médicos nuevos" (M1). Consideraron fundamental tener oportunidades para diversificar sus actividades en beneficio de los pacientes y la Salud Pública del país. Según refirieron, uno de los elementos de mayor desmotivación sería la monotonía, generada por la sobrecarga asistencial, hecho que no permite otorgar una atención de calidad ni desarrollar iniciativas en sus respectivos establecimientos: "no quiero hacer sólo morbilidad, me he capacitado y puedo hacer otras cosas" (M6), "la monotonía es un problema para ellos y nos lo han hecho saber" (D2), "los directivos cometemos errores en darles sólo asistencialidad" (D1).

\section{5.- Rol de las escuelas de medicina en la elección por APS}

Se indagó dirigidamente por la formación de pregrado en APS. Todos fueron enfáticos en señalar que ésta estaba orientada al desarrollo de competencias clínicas intrahospitalarias y, por lo tanto, no había sido relevante en su elección laboral: "la universidad no motiva ni llama a ser aporte en APS" (M1), "la universidad no influyó en mi elección en APS" (M2), "dado que las rotaciones son mínimas, no alcanzas a valorarla" (M2). Se refirieron favorablemente a los cambios curriculares en la formación médica, a pesar de que aún falta evaluar su impacto en las nuevas generaciones de egresados: "en mis tiempos, el mensaje era hacer especialidad, los médicos nuevos saben que existe la salud familiar en APS" (M7), "aquí pasan los alumnos de medicina, pero es poco el tiempo y no alcanzan a empaparse de nuestro rol en la salud" (D2).

\section{DISCUSIÓN}

En nuestro país, Chile, se estima un déficit de médicos en APS cercano al 50\%. Publicaciones recientes refieren que en consultorios municipalizados sólo la tercera parte de los profesionales tendría una antigüedad superior a tres años ${ }^{24}$. En coincidencia, en la muestra analizada los médicos chilenos realizaban un tercio de las horas médicas totales y sólo la mitad de estas horas eran ejercidas por profesionales de mayor antigüedad laboral. 
La información obtenida nos ilustra respecto a la falta de una política de recursos humanos que motive el reclutamiento y estabilidad del médico en $\mathrm{APS}^{25}$, a pesar de ser éste el elemento central en el Plan de Acción propuesto para las Américas ${ }^{26}$ y de las intenciones políticas al respecto en nuestro país ${ }^{27}$. Por esta razón, es fundamental identificar los elementos que motivan al médico a desarrollar su carrera profesional en este nivel de atención.

Analizamos las características biodemográficas y laborales de los médicos de mayor antigüedad laboral, encontrando un grupo de mayor edad, pero sin diferencias con los más nuevos en las demás variables sociodemográficas y laborales estudiadas.

Al indagar en las motivaciones extrínsecas desatacó el descontento por las bajas remuneraciones, falta de contratos estables y percepción de prejuicio por parte de los pares. En cuanto a las motivaciones intrínsecas, la carrera profesional ha sido ampliamente considerada como un incentivo para el desarrollo profesional ${ }^{28}$ y estabilidad laboral. No obstante, los entrevistados refirieron obstáculos para su desarrollo profesional y falta de interés por seguir una carrera funcionaria, a pesar de haber ejercido cargos directivos. A partir de las respuestas obtenidas se puede inferir que estos factores son elementos desmotivadores para el reclutamiento y estabilidad laboral en APS.

Entonces, al tratar de comprender quiénes son esos médicos que eligen la APS, a pesar de no contar con las condiciones laborales idóneas, nos encontramos con un grupo de médicos motivados fundamentalmente por factores trascendentes, es decir, por el deseo de dejar algo más allá en su trabajo diario, destacando el compromiso vocacional con la salud pública, la relación médico- paciente y la confianza mutua ${ }^{29-32}$. Llama la atención que el trabajo en equipo, elemento destacado en la literatura ${ }^{33}$, no fuera mencionado.

Se atribuyó especial valoración a la atención integral, con énfasis biopsicosocial, no sólo en el aspecto curativo sino también promocional y preventivo, acorde a la filosofía de la reforma de salud ${ }^{3}$. No obstante, las actuales políticas de los establecimientos de salud privilegian una práctica médica centrada en la satisfacción de necesidades curativas y asistenciales, en desmedro de las motivaciones descritas.

Las inadecuadas condiciones laborales, según la percepción de los médicos chilenos, se han traducido en que la APS haya sido copada por médicos extranjeros, la mayoría de acuerdo a convenios bilaterales que no exigen evaluación de competencias del profesional ${ }^{34-35}$.
Hay que tener presente que el actual escenario podría transformarse drásticamente en los próximos años, producto del explosivo aumento de Escuelas de Medicina ${ }^{36}$ con una formación orientada hacia la APS ${ }^{37}$. Ha sido ampliamente discutido el rol de las Facultades de Medicina, y la orientación de sus políticas y currículos, en la elección de las especialidades médicas $^{32}$. Según refirieron los entrevistados, la formación recibida por ellos estaba centrada en el logro de competencias clínicas intrahospitalarias, marcando de esta manera una clara brecha con los requerimientos para el ejercicio profesional posterior, tal como se ha reportado en programas de formación de especialistas en el país ${ }^{38}$.

A partir de la teoría motivacional de Pérez-López20, $\mathrm{Simo}^{39}$ analiza la vinculación que desarrolla el médico familiar con la organización de salud y propone estrategias para enfrentarlo. Cuando las motivaciones trascendentales predominan sobre las demás, como ocurrió con nuestros entrevistados, las políticas de los establecimientos de salud deberían potenciarlos haciéndolos sentir parte de un proceso en el logro de los objetivos de la organización. Estudios revelan que los profesionales de la salud están ávidos de participación en sus centros, de aportar ideas y ser reconocidos en sus esfuerzos ${ }^{40}$.

A pesar de que los médicos de mayor estabilidad laboral refirieron que no está en sus planes abandonar la APS, propusieron privilegiar estrategias para combatir la monotonía del trabajo diario y tener oportunidades para mejorar la calidad e integralidad de su atención, debiendo superarse los obstáculos percibidos por parte de los directivos.

Se enfatizó la necesidad de fomentar las oportunidades de capacitación y desarrollo profesional, fundamentalmente como estrategia de reclutamiento de nuevos médicos en APS ${ }^{41}$. En ese sentido están orientadas las políticas del Ministerio de Salud, que ha generado recientemente programas de especialidades básicas para médicos que trabajan en aquellas zonas menos dotadas ${ }^{42}$.

Este estudio constituye una primera aproximación al conocimiento de las motivaciones que llevan al médico chileno a trabajar en forma estable en un Servicio de Salud urbano, de la Región Metropolitana. Aquellos médicos que optan por la APS para el desarrollo profesional estarían motivados fundamentalmente por factores trascendentes, como el compromiso vocacional, la relación entablada con la comunidad y con sus pacientes y el compromiso adquirido con la salud pública. Se plantea la relevancia que los esta- 
blecimientos de salud tengan del trabajo médico, una visión más holística que la pura asistencialidad y la resolución de contingencias, como modo de fomentar estrategias que permitan al profesional trascender e impactar directamente en la salud de la población.

\section{AGRADECIMIENTOS}

A Teresa Millán y Ruth Depaux por su valioso aporte a este estudio, entregando su experiencia en atención primaria y salud pública de nuestro país. También agradecemos a Ana Dovoredsky por su revisión y comentarios.

\section{BIBLIOGRAFÍA}

1. OMS y UNICEF, Alma Ata 1978, Atención Primaria de Salud. Ginebra: OMS; 1978.

2. Montoya-Aguilar C. Atención Primaria de salud, Alma Ata otra vez y la experiencia en Chile. Cuad Med Soc (Chile) 2008; 47(3):147-54

3. Ministerio de Salud, Gobierno de Chile. Objetivos sanitarios y modelo de salud para la década 2000 - 2010. Documento oficial. Enero 2002

4. Montoya-Aguilar C. Médicos en el nivel primario del sistema público de atención de salud de Chile: tendencia, distribución geográfica y normas. Cuad Med Soc (Chile) 2008; 48(4):21525.

5. Cámara de diputados. NOTICIAS. Presentan propuesta para superar déficit de médicos en atención primaria. [Citado 21/10/2008]. Disponible en: http://www.camara.cl/diario/noticia.asp?vid=20085

6. AgrupacióndeMédicosdeAtenciónPrimaria.Conclusionesmesa de trabajo: Escenario actual de los médicos de atención primaria de salud. 2 de septiembre 2006. [Citado 20/07/2006]. Disponible en: http://www.médicosaps.cl/Documentos/Conclusiones\%20Mesa\%20Condiciones\%20Trabajo\%20Médicos\%20 APS.pdf

7. Jiménez de la Jara J. Estrategias de los médicos para hacer frente a la crisis de la profesión. Rev Med Chile 2005; 133(6):707-12.

8. Jiménez de la Jara J. Cambios en el entorno del trabajo médico. Rev Med Chile 2004; 132(5):637-42.

9. Katerndahl D, Parchman M, Wood R. Perceived complexity of care, perceived autonomy, and career satisfaction among primary care physicians. J Am Board Fam Med 2009; 22(1):2433.

10. Sibbald B, Enzer I, Cooper C, Rout U, Sutherland V. GP job satisfaction in 1987, 1990 and 1998: lesson for the future? Family Practice 2000; 17(5):364-71.

11. Lambert J, Goldacre M. Recruitment of UK trained doctors into general practice: finding from national cohort studies. $\mathrm{Br} \mathrm{J}$ Gen Pract. 2002; 52:364 -72.

12. Sobreques J, Cebria J, Segura J, Rodríguez C, García M, Juncosa S. La satisfacción laboral y el desgaste profesional de los médicos de atención primaria. Aten Primaria 2003; 31(4):22733.

13. González Benedetti A, Segovia Dreyer I. La medicina General Familiar en Chile. Cuad Med Soc (Chile) 1995; 36( $n^{\circ}$ extraordinario):29-33.
14. Goic A, Armas R. Descentralización en salud y educación: La experiencia chilena. Rev med Chile 2003; 131(7):788-98.

15. Vergara M. La renta medica en el sector público de salud. Revista Chilena de Salud Publica 2001; 5(2-3):102-10.

16. Horwitz N. El cambio de la práctica Médica. Desafíos psicosociales. Rev Med Chile 2004; 132:768-72.

17. Newton D, Garayson M, Thompson L. The variables influence of lifestyle and income on medical students'career specialty choices: data from two U.S. Medical Schools, 1998-2004. Academic Medicine 2005; 80(9):809-14.

18. Solimano G, Pepper S. Perspectivas de la red asistencial de salud en Chile. Santiago de Chile: CORSAPS; 1999.

19. Maslow AH. Motivation and Personality. New York; Harper \& Row; 1954.

20. Pérez López, JA. "Las Motivaciones Humanas". Barcelona: Instituto de Estudios de superiores de la Empresa, Universidad de Navarra; 1987.

21. Documento del SSMC: Unidad de Control de Gestión y Estadística, Memorandum $n^{\circ} 52$ del 17 de Noviembre 2006. Fuente INE - MINSAL 2002.

22. Patton M. Qualitative Evaluation and Research Methods. United States of America: Sage publications; 1990.

23. Crabtree B, Miller W. Doing qualitative Research. United States of America: SAGE publications; 1992.

24. Ipinza M. Cómo solucionar el Déficit de Médicos en los Consultorios Urbanos Municipalizados. Cuad Med Soc (Chile) 2004; 44(2):73-80.

25. Castro JL. El Déficit de Médicos en la Atención Primaria Urbana. Cuad Med Soc (Chile) 2005; 45(4):241-2.

26. Consulta regional sobre la renovación de la atención primaria de salud (APS) en las Américas OPS/ OMS. Montevideo, Uruguay. Julio 2005. [Consultado 8/07/09]. Disponible en: http:// www.paho.org/spanish/AD/THS/OS/APS-agenda.pdf

27. Rodríguez P. Superar la crisis en Salud Pública: Desafío Nacional. Cuad Med Soc (Chile) 2008; 48(3):145-6.

28. Gervas J. La Carrera Profesional desde el punto de vista de un Médico Clínico. Rev Clin Med Fam [online] 2008; 2(4):167-170 [Consultado15/06/09]. Disponible en: http:// scielo.isciii.es/scielo.php?script=sci_arttext\&pid=S1699-695X2008000200006\&lng=es\&nrm=iso

29. Gómez Esteban R. La relación médico-paciente, efectos sobre la salud del médico. Psiquis 2003; 24(2):46-58.

30. Zuger A. Dissatisfaction with medical practice. N Engl J Med 2004; 350(1):69-75.

31. Bascuñan M L. Cambios en la relación médico-paciente y nivel de satisfacción de los médicos. Rev Med Chile 2005; 133(1):11-6.

32. Escobar F, López-Torres J. ¿Qué determina la elección de una Especialidad en Medicina? Rev Clin Med Fam [online]. 2008; 2(5):216-25 [citado 2009-06-14]. Disponible en: http:// scielo.isciii.es/scielo.php?script=sci_arttext\&pid=S1699-695X2008000300005\&lng=es\&nrm=iso

33. Muñoz E, Linares L, Torrent M, Coll JM. Influencia del clima laboral en la satisfacción de los profesionales sanitarios. Aten Primaria 2006; 37(4):209-14.

34. Enríquez O, Mena B. Habilitación profesional. Condiciones para el aseguramiento de la calidad de la educación médica y condiciones para la confianza recíproca. Experiencia y visión de ASOFAMECH. Rev Med Chile 2005; 133(4):483-94.

35. Goic A. Disponibilidad de médicos en Chile y su proyección a mediano plazo. Rev Med Chile 1994; 122:141-53. 
36. MINEDUC. [Consultado 20/07/2008]. Disponible en: http://directorio.educador.cl/oferta_2004/busqueda_carrera.php

37. Goic A. Seminario sobre formación de médicos en la actualidad en Chile. Rev Med Chile 2003; 131(2):209-12.

38. Schonhaut L, Millán T, Hanne C. Formación de especialistas en pediatría y su adecuación a la realidad epidemiológica y laboral. Rev Chil Pediatr 2007; 78(6):599-606.

39. Simo Miñana J, Chinchilla Albiol N. Motivación y médicos de familia (I). Aten Primaria 2001; 28(7):484-90.
40. Jubete Vázquez MT, Lacalle Rodríguez-Labajo MT, Fuertes M, Cortes Rubio JA, Ruiz M. Riesgo de la calidad de vida profesional en los trabajadores de atención primaria del Área 1 de Madrid. Aten Primaria 2005; 36(2):112 -4.

41. Román A, Pineda R, Senoret S. Perfil y número de médicos generales que requiere el país. Rev Méd Chile 2007; 135(9):1209-15.

42. Montoya C, Ipinza M. "Un programa para recuperar y mejorar el aporte de los médicos en la Atención Primaria de Salud" Cuad Med Soc (Chile) 2009; 49(1):16-25. 